\title{
CYSTICERCUS LONGICOLLIS CHEZ DES CANIDÉS
}

\author{
Par J. CALLOT et G. GAYOT
}

Il paraît oiseux de parler encore de Cysticercus longicollis Rud. qui a été décrit ou étudié morphologiquement et biologiquement par de nombreux Helminthologistes : Bremser, Tschudi, Leuckart, Braun, Bott, Gläser, Joyeux, pour ne citer que les principaux. Mais cette curieuse forme larvaire n'a été encore signalée jusqu'à présent que chez des Rongeurs et la Taupe, à part une exception sur laquelle nous reviendrons plus loin. Aussi sa découverte chez un Fennec et les constatations que nous avons pu faire à ce propos nous ont-elles paru mériter d'être rapportées.

$$
* *
$$

Un Fennec (Fennecus zerda), originaire du Maroc, mais en captivité en France depuis deux ans, présente en juin 1958 une tuméfaction de la région cervicale. Une exploration, pratiquée par un confrère vétérinaire au mois de novembre suivant, montre qu'il s'agit de "cysticerques ».

L'animal est de nouveau examiné le 25 novembre et l'on décide de l'opérer ; mais il meurt sous l'effet de l'anesthésie. Il est autopsié au Laboratoire de Parasitologie où le cadavre avait été immédiatement apporté.

La région thoracique antérieure est entièrement déformée comme par un manchon entourant l'animal. L'incision sur la ligne sternale donne issue à une prodigieuse quantité de vésicules vermineuses, qu'une exploration rapide montre provenir de poches irrégulières s'insinuant dans toutes les directions, entre les plans musculaires.

Cette parasitose s'étend de la base du cou à la base du thorax. Aucune vésicule ne se trouve dans la cavité pleurale ou péritonéaí, ni dans la boitte crânienne, ni même aux membres ou dans la paroi abdominale (1). Il n'y a pas de Cestodes intestinaux.

(1) La région axillaire est souvent citée comme siège de $C$. longicollis, mais on l'a trouvé aussi dans les cavités splanchiques. 
On cherche à isoler par dissection de grandes poches que l'on voit saillir sous les aponévroses, les muscles, l'omoplate, mais il est impossible de le faire sans que des vésicules ne se répandent immédiatement, et ce n'est qu'en pratiquant des prélèvements considérables que l'on arrive à en fixer in toto, entourées de muscles et d'aponévroses.

Les vésicules qui s'échappent ainsi des incisions sont transparentes, généralement ovoïdes; beaucoup sont marquées à un pôle par une tache blanchâtre (1). Leur taille est très variée, de $1 \mathrm{~mm}$. à $2 \mathrm{~cm}$. Si beaucoup sont ovoïdes ou subsphériques, certaines sont munies d'expansions. Elles sont légèrement mobiles.

L'examen rapide montre l'existence à un des pôles, dans la tache blanche, d'un'scolex invaginé, qui s'évagine très facilement sous l'effet de la pression et même spontanément dans les liquides physiologiques, mais, outre ce scolex principal, plusieurs de ces vésicules contiennent deux ou trois scolex à leur intérieur, comme des cénures.

De plus, des vésicules, les plus grandes en général, donnent naissance, au pôle opposé au scolex, à d'autres vésicules, plus petites, et qui sont rattachées par un fin pédicule à la vésicule mère et sont, de toute évidence, à l'origine des centaines de petites vésicules que l'on rencontre dans la région périthoracique.

Des vésicules sont prélevées et fixées par différents procédés; d'autres ont été introduites dans la cavité péritonéale ou dans les espaces celluleux de la région thoracique du lapin; enfin, un certain nombre ont été conservées vivantes dans des solutions nutritives.

$$
*
$$

L'examen des masses parasitaires fixées in toto a montré deux types de cavités emplies par des vésicules :

A) Un premier type est constitué par des poches dont la paroi est parsemée de petits nodules blanchâtres, se détachant facilement, qui sont des scolex ; mais, à la dissection, on ne peut séparer des tissus de l'hôte une membrane parasitaire, comme on le fait si facilement avec les Cénures du type sérial. Ceci s'explique à l'examen microscopique qui montre que la paroi des poches est constituée uniquement par une réaction granulomateuse considérable et en pleine évolution. On a l'impression, au premier coup d'œil, que

(1) Certains auteurs ont comparé le flot de vésicules à un « tapioca », à du frai, et comme nous, ont pu les recueillir, vu leur abondance, « à la cuiller ». 
des scolex sont insérés directement sur cette membrane réactionnelle, mais, en réalité, il s'agit de scolex envahis et fixés par la réaction de l'hôte.

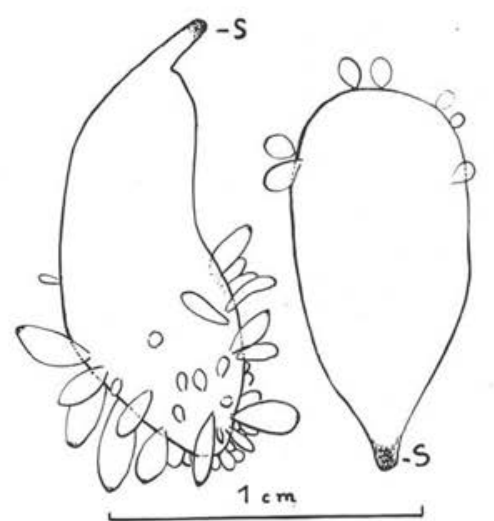

Fig. 1. - Vésicule portant des bourgeons. S, scolex.

B) Dans un second type, on voit encore une membrane réactionnelle, mais plus mince et plus au repos, semble-t-il, limitant une cavité où se trouvent des vésicules qui paraissent parfaitement intactes, quoique déformées par l'action des agents fixateurs.

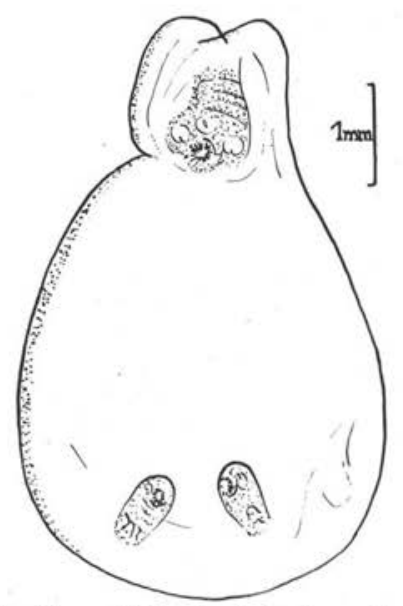

Fig, 2, - Vésicule à trois scolex.

L'examen des vésicules confirme ce qui avait été vu à l'inspection première. A partir d'une certaine taille $(0,5 \mathrm{~cm}$. environ), elles 
contiennent toujours un scolex polaire très développé, dont nous examinerons plus loin les caractéristiques, et souvent un ou deux plus petits intra-vésiculaires. Les vésicules filles exogènes, encore rattachées, ne contiennent pas de scolex, mais son ébauche peut se voir sur les plus grosses au pôle opposé au pédicule. Ce pédicule est, le plus souvent, très fin, et ceci quelle que soit la taille de la vésicule fille. Cette prolifération est donc morphologiquement très différente de ce qu'on voit dans les cénures qui sont bordés de digitations «en franges » très caractéristiques.

Les vésicules filles peuvent être au nombre de quelques unités ou de plusieurs dizaines à des stades variés de développement; on trouve des vésicules libres qui ne sont pas plus grosses que celles qui sont encore fixées et qui ont dû se séparer, soit du vivant de l'animal, soit au cours de manipulations.

Les crochets du scolex sont au nombre de 16 ou 17 paires de grands et petits crochets et sont du type ténia, mais ils sont remarquables par !e développement de la lame (1).

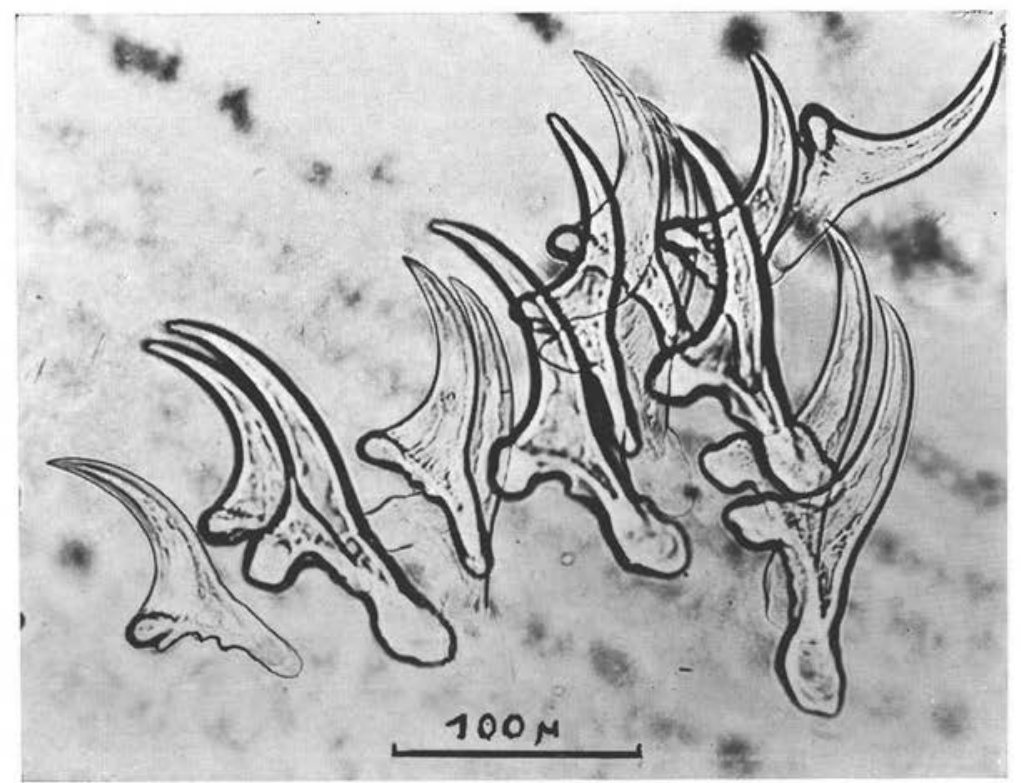

Fig. 3. - Grands et petits crochets rostraux.

(1) A titre de comparaison nous voyons que si l'on prend pour 100 la distance $b f$, on a pour $a b$ chez le Cénure sérial 133, alors que pour $C$. longicollis du Fennec on a 80.

Avec la terminologie de Clapham et Peters, en mesurant la lame de son origine $\mathrm{E}$ à son extrémité $\mathrm{A}$, on a pour le Cénure sérial $60,02 \mu(\mathrm{mx} .62, \mathrm{~min}, 60)$ et pour le Cystique du Fennec $94 \mu$ (max, 97, min. 87). 
Les mensurations, effectuées d'après les indications de Meggitt, donnent les chiffres suivants pour les grands crochets :

\begin{tabular}{|c|c|c|c|c|c|c|}
\hline & al & $a c$ & $f c$ & $e d$ & $a b$ & Long. totale \\
\hline & - & - & - & - & - & - \\
\hline Moyenne .... & $74,3 u$ & $160,5 \mu$ & $68 \mu$ & $77 \mu$ & $85,9 \mu$. & $170 \mu$. \\
\hline$\ldots \ldots \ldots$ & 85 & 170 & 80 & 90 & 90 & 190 \\
\hline Min. . ........ & 65 & 150 & 60 & 65 & 75 & 165 \\
\hline
\end{tabular}

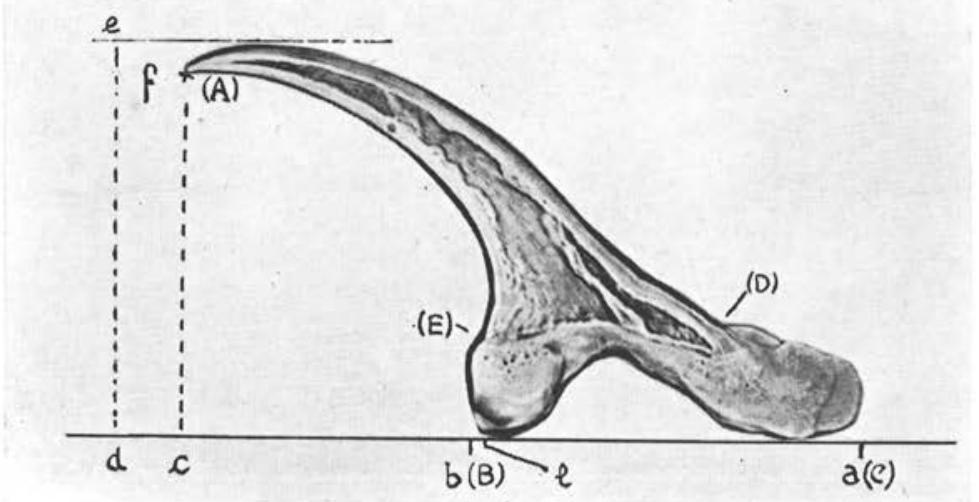

Fic. 4. - Grand crochet du rostre. $a, b, c, d, e, l$ : points de Meggitt. A, B, C, D, E : points de Clapham et Peters.

La lame des petits crochets est aussi relativement développée et le manche porte du côté concave une ou deux saillies très nettes ; la garde, sur quelques exemplaires seulement, parait bilobée; les mensurations pour ces petits crochets sont :

\begin{tabular}{|c|c|c|c|c|c|}
\hline & al & $a c$ & $f c$ & ed & long. totale \\
\hline & 一 & 一 & 一 & 一 & - \\
\hline Moyenne... & $59 \mu$ & $111 \mu$ & $61 \mu$ & $68 \mu$ & $140 \mu$. \\
\hline Max. ....... & 70 & 120 & 65 & 70 & 150 \\
\hline$\ldots \ldots$ & 50 & 95 & 52 & 65 & 130 \\
\hline
\end{tabular}

Nous avions done affaire à un Cysticerque très particulier, tant par l'abondance des vésicules, par leur répartition, par le caractère presque malin de leur envahissement, par leur bourgeonnement interne et externe, par la morphologie des crochets, que par le fait de le trouver chez un Canidé, 
A vrai dire, il existe une observation absolument superposable à la nòtre d'un Cysticerque bourgeonnant chez un Renard, c'est celle du Cysticercus multiformis, décrit par Hölldobler chez un Renard d'Allemagne, et nous n'hésitons pas à l'identifier à celui du Fennec (1).

Mais, en réalité, dans l'un comme dans l'autre cas, il ne peut s'agir que de Cysticercus longicollis, c'est-à-dire de la forme larvaire du Taenia crassiceps Rud. du Renard.

Les nombreuses études de cette curieuse forme larvaire chez les Rongeurs ou chez la Taupe, et en particulier les dessins de Bott, rappellent tout à fait ce que nous avons constaté. L'aspect très particulier des crochets, avec leur lame si développée, leur taille, est absolument caractéristique de la larve de Taenia crassiceps (2).

$$
* *
$$

Nous avons dit plus haut que des vésicules avaient été inoculées à des lapins dans les espaces celluleux de la région scapulaire et dans le péritoine. Dans ces lapins sacrifiés et autopsiés, on n'a retrouvé aucune trace du matériel greffé.

Des vésicules, prélevées aseptiquement au cours de l'autopsie du Fennec, ont été réparties dans des tubes contenant un liquide nutritif destiné à la culture de cellules humaines (2), et conservées à l'étuve à $37^{\circ}$. Le milieu, qui est renouvelé deux fois par semaine, ne montre aucune trace de souillures pendant la durée de l'expérience.

Certains cystiques sont demeurés vivants dans ce milieu pendant 39 jours. Les scolex se sont dévaginés et sont animés de mouvements très vifs, mais les vésicules les plus petites n'ont montré aucune trace de développement, se sont rétractées et sont mortes. Les vésicules pourvues d'un scolex évaginé se sont rétractées peu à peu ; mais quelques scolex ont présenté au bout de trois semaines environ une manière de vésiculisation avec formation d'une néovésicule parfois allongée aux dépens de la région cervicale. Ceci rappelle les constatations de Henry et Ciuca (1914), ou de Coutelen sur le Cénure sérial dont le scolex se vésiculise à partir du pédoncule pour former des vésicules endogènes.

(1) Malheureusement, la description d'Hölldobler est fort incomplète en ce qui concerne les crochets. Mais les figures des vésicules sont absolument identiques à ce que nous avons vu.

(2) Ils sont très bien représentés sur les planches du mémoire de Gläser.

(3) Adaptation du liquide décrit par P. Lépine et coll. Ann, Inst. Pasteur, $1956,90,654$. 
L'analogie ne doit pas être poussée plus loin, car nous n'avons pas l'impression qu'il y ait formation de vésicules endogènes chez C. longicollis, tout au moins dans les limites de nos observations.

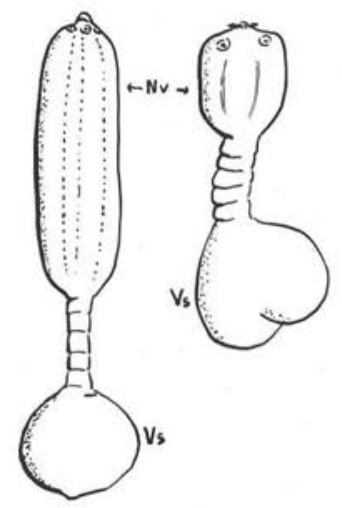

FIg. 5. - Scolex en culture. Vs, vésicule primitive rétractée ; $\mathrm{Nv}$, vésiculisation du scolex.

$$
* *
$$

\section{Discussion}

La présence chez l'hôte définitif (ou un hôte très voisin) de la forme larvaire d'un Cestode n'est pas un fait exceptionnel, et le plus bel exemple en est donné par les cas de ladrerie humaine à C. cellulosæ. Les ténias habituels des Canidés ont rarement été signalés à l'état larvaire chez ces hôtes. Cependant, Joyeux et Baer (1936, p. 438) citent le chien comme hôte, certes exceptionnel, du C. serialis, de même qu'on aurait trouvé des hydatides aussi chez des chiens.

On doit aussi se demander comment un Fennec en captivité depuis longtemps a pu être contaminé. Il est possible qu'il ait été porteur lui-même d'un Taenia crassiceps, en supposant que ce ténia, qui semble peu ubiquiste et très spécifique du Renard, ait pu évoluer chez lui. Mais cela ne fait que repousser, sans le résoudre, le problème des rapports entre un Fennec captif et un Renard... 


\section{RÉSU MÉ}

$1^{\circ}$ Nous signalons la présence, chez un Fennec, en captivité depuis deux ans à Strasbourg, d'une Cysticercose envahissante due à la prolifération du Cysticercus longicollis, forme larvaire de Tania crassiceps du Renard.

$2^{\circ}$ Le Cysticercus multiformis de Hölldobler (1937) doit être identique à cette forme larvaire.

\section{BiBLIOGRAPHIE}

Braun (M.), 1896. - Helminthologische Notizen. V. Ein proliferierender Cysticercus und die zugehörige Tänie. Centr. f. Bakt., 1, XX, p. 580-588.

Bотт (A.), 1898. - Ueber einen durch Knospung sich vermehrenden Cysticercus aus dem Maulwurf. Zeits. Wiss. Zool., LXIII, p. 115-140, pl. VI et VII.

Clapham (P. A.) et Peters (B. G.), 1941. - Differentiation of Coenurus species by hook measurements. Jl Helminth., XIX, p. 75-84.

GläSEr (H.), 1909. - Zur Entwicklungsgeschichte des Cysticercus longicollis Rud. Zeits. Wiss. Zool., XCII, p. 540-561, pl. XVI et XVII.

Hölldobler (K.), 1937. - Cysticercus multiformis nov. spez., eine noch nicht beschriebene Finnenform einer Cyclophyllidea. Zeits. Parasitenk., IX, p. $523-528$.

Joyeux (Ch.), 1927. - Deux parasites nouveaux pour la marmotte des Alpes : Marmota marmotta L. (cysticerque et sarcosporidie). Ann. parasitol., V, p. 381-382.

Joyeux (Ch.) et Baer (J. G.), 1936. - Cestodes. Faune de France, fasc. 30.

WARDLE (R. A.) et Mc Leod (J. A.), 1952. - The Zoology of the Tapeworms. Minneapolis, M.M.

(Institut de Parasitologie, Fac. Méd. et Laboratoire

Vétérinaire départemental, Strasbourg). 